\title{
Percutaneous Arterial Gene Transfer in a Rabbit Model Efficiency in Normal and Balloon-dilated Atherosclerotic Arteries
}

\author{
Guy Leclerc, Dov Gal, Satoshi Takeshita, Sigrid Nikol, Lawrence Weir, and Jeffrey M. Isner \\ Departments of Medicine (Cardiology), Pathology, and Biomedical Research, St. Elizabeth's Hospital, \\ Tufts University School of Medicine, Boston, Massachusetts 02135
}

\begin{abstract}
The possibility of using an exclusively percutaneous strategy to deliver foreign DNA to normal and balloon-dilated atherosclerotic arteries was studied by analysis of transfection efficiency in a rabbit model. A total of 22 external iliac arteries from 22 rabbits (10 normal and 12 atherosclerotic) were transfected with a solution of luciferase expression vector plasmid and liposome, using a dual balloon-catheter system. Analysis of the transfected segments revealed luciferase activity in 10 of the 22 arteries (4/10 normal vs $6 / 12$ balloon-injured atherosclerotic, $P=$ NS); no activity could be detected in the contralateral limb arterial segments used as controls. Luciferase activity levels in successfully transfected segments measured $4.10 \pm 1.19$ ( $m \pm$ SEM) Turner light units (TLU), with 3.03 \pm 1.16 TLU found in normals vs $4.81 \pm 1.87 \mathrm{TLU}$ in balloon-injured atherosclerotic arteries ( $P=\mathrm{NS})$. In situ hybridization of successfully transfected atherosclerotic sections showed expression of the luciferase gene mRNA from rare cells $(<1 / 1,000)$ limited to the neointimal lesion. Thus, expression of new genetic material may be achieved in both normal and balloon-dilated atherosclerotic arteries following an exclusively percutaneous approach. The low efficiency of the current delivery strategy, however, represents a potential limitation that must be improved if this strategy is to be applied as a therapeutic approach to human vascular disease. ( J. Clin. Invest. 1992. 90:936-944.) Key words: angioplasty $\bullet$ atherosclerosis $\bullet$ gene expression $\bullet$ intimal proliferation $\cdot$ luciferase
\end{abstract}

\section{Introduction}

The ability to perform site-specific transfer of genetic material in vivo has important implications, both fundamental and therapeutic. As emphasized previously (1), a system for in vivo gene transfer constitutes a unique opportunity to study gene regulation under pathological conditions that cannot be altogether reproduced in vitro. Moreover, rapid advances in recombinant DNA technologies have permitted the initiation of several human protocols for the treatment of specific pathologies $(2,3)$. Although gene therapy presently targets single gene dis-

This work was presented in part at the 64th Annual Scientific Sessions of the American Heart Association, Anaheim, CA, 11-14 November 1991.

Address correspondence to Jeffrey M. Isner, M. D., St. Elizabeth's Hospital, 736 Cambridge Street, Boston, MA 02135.

Received for publication 30 September 1991 and in revised form 10 March 1992.

J. Clin. Invest.

(C) The American Society for Clinical Investigation, Inc. 0021-9738/92/09/0936/09 \$2.00

Volume 90, September 1992, 936-944 orders, its concept may well expand into the field of disease prevention (4).

From the standpoint of vascular disorders, gene therapy represents a potential prevention strategy for restenosis, a phenomenon that has resisted a plethora of conventional and interventional approaches (5). Seminal reports by previous investigators have demonstrated that successful expression of newly introduced genetic material in vascular tissues can be accomplished in vivo. These previous studies, carried out in surgically exposed arteries of normal dogs and swine (6-10), have employed both direct and indirect means of gene transfer. The former involved direct transfer of genetic material into an intact arterial wall using a retroviral vector (6) or cationic liposomes $(6,7)$ as the delivery vehicle. The latter approach involved the use of cells which were genetically engineered in vitro, and subsequently introduced into the vasculature $(8,9)$ or implanted into arterial prosthetic grafts (10). Clinical implementation of gene therapy for restenosis would be most conveniently performed via an exclusively percutaneous approach to achieve site-specific delivery of the new genetic material. To realize this goal, at least three requirements must be fulfilled. First, it must be shown that gene transfer can be achieved using an exclusively percutaneous approach. Second, because such attempts to preempt restenosis would likely be performed in atherosclerotic arteries after percutaneous revascularization, it must be demonstrated that gene transfer can be achieved under circumstances representing the combined effects of these two variables, namely, atherosclerotic lesions treated by balloon angioplasty. Third, it must be established that gene transfer can be efficiently performed, both in terms of the frequency of successful deliveries, as well as the resulting levels of gene expression.

Accordingly, this study was designed to evaluate the use of an exclusively percutaneous strategy to deliver foreign DNA to normal and balloon-dilated atherosclerotic arteries in a rabbit model. Using a dual balloon-catheter system, we instrumented 22 animals, of which 10 were normal and 12 had experimentally induced atherosclerosis; in each case, one external iliac artery was transfected with a solution of luciferase expression vector plasmid and liposomes. To provide more direct information regarding the extent of transfection in the diseased arteries, in situ hybridization was performed on successfully transfected atherosclerotic arterial sections.

\section{Methods}

DNA-liposome solution. We used the firefly luciferase gene as the reporter to monitor the results of our in vivo transfection procedures. The mammalian expression vector, pRSVLUC (courtesy of Dr. Allen Brasier, Massachusetts General Hospital, Boston, MA), consisted of a full length firefly Photinus pyralis luciferase cDNA (pJD 204) (11) inserted into the plasmid pGEM3 (Promega Corp., Madison WI)(12), under the control of the Rous sarcoma virus long terminal repeat pro- 


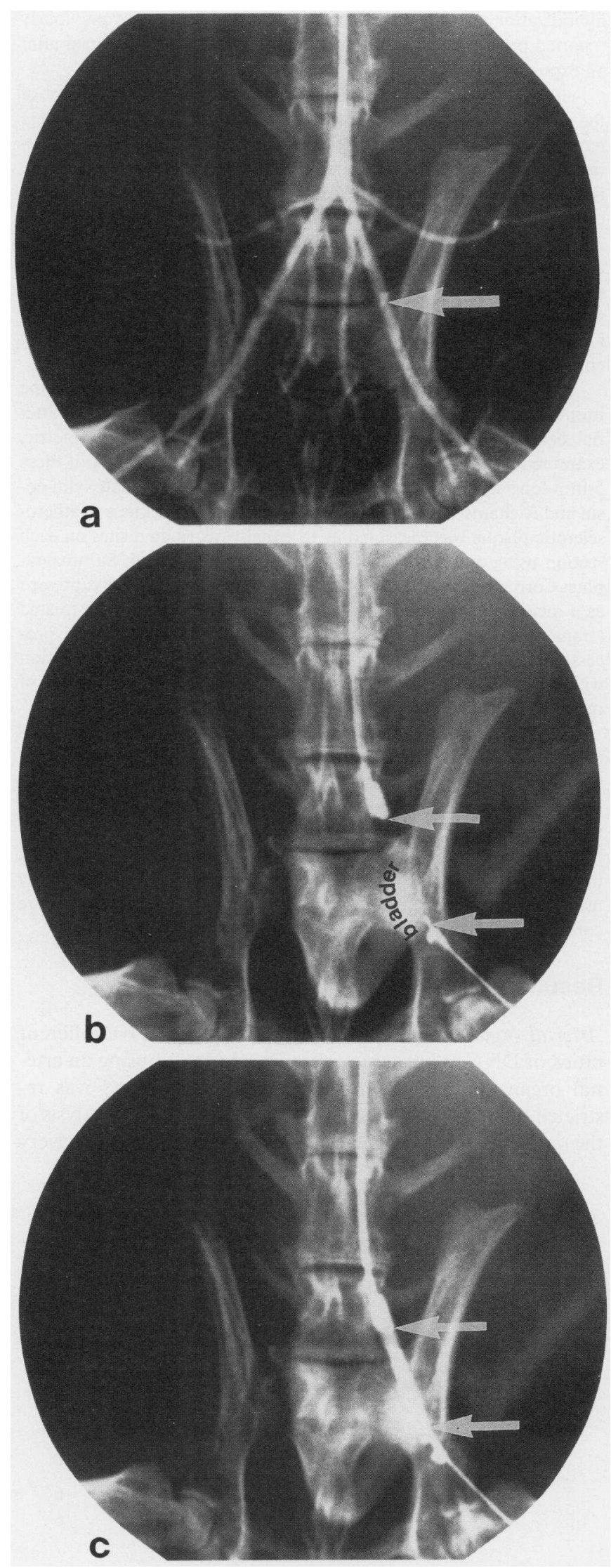

Figure 1. Radiographic recording of distal aortic angiogram of normal rabbit and dual balloon-catheter system used to perform percutaneous intraarterial transfection under fluoroscopic guidance. ( $a$ ) Distal aortic angiogram and peripheral vasculature. Arrow indicates left exter- moter. The DNA-liposome solution was prepared within the $15 \mathrm{~min}$ before the transfection procedure. A total of $30 \mu \mathrm{g}$ of DNA and $90 \mu \mathrm{g}$ of cationic liposomes ( $N$-[1-(2,3-dioleyloxy)propyl]- $N, N, N$-trimethylammonium chloride, Lipofectin; Bethesda Research Laboratories, Gaithersburg, MD) $(13,14)$ were added to reduced-serum medium (Opti-MEM; Gibco Laboratories, Grand Island, NY) to a final vol of 1 $\mathrm{ml}$, at room temperature, in a sterile polystyrene tube. To verify the efficiency of the DNA-liposome transfection solution in vascular tissue, we transfected rabbit thoracic arterial segments cultured in vitro using a method adapted from those previously described $(15,16)$. Briefly, thoracic aortas freed of adherent tissues were cut to 3-mm-wide segments and washed in sterile PBS. They were then incubated at $37^{\circ} \mathrm{C}$ in a humid atmosphere of $95 \%$ air and $5 \% \mathrm{CO}_{2}$, with shaking, for 30 min with $2 \mathrm{ml}$ of Opti-MEM, $30 \mu \mathrm{g}$ of pRSVLUC plasmid and varying amounts of Lipofectin to obtain DNA-liposomes ratios from 1:1 to 1:5 (wt/wt). At least six arterial segment transfections were performed for each ratio tested. Following the transfection period, media was changed to Dulbecco's minimal Eagle's modified medium (Gibco) supplemented with $20 \%$ fetal calf serum (Gibco), $2 \mathrm{mM}$ glutamine (Gibco), $100 \mathrm{IU} / \mathrm{ml}$ penicillin, and $100 \mu \mathrm{g} / \mathrm{ml}$ streptomycin (Gibco). These conditions were maintained for $3 \mathrm{~d}$ and then the specimens were analyzed for luciferase expression (vide infra).

In vivo percutaneous transfection procedure (Fig. 1). New Zealand white rabbits (Pine Acre Rabbitry, Norwood, MA) were used for this study. They were housed and handled according to a protocol approved by St. Elizabeth's Institutional Animal Care and Use Committee. Lesions in the atherosclerotic animals were induced by a hypercholesterolemic diet and balloon endothelial denudation of the peripheral vasculature according to previously reported protocols (17).

Transfections were performed in the external iliac arteries of the anesthetized animals using a dual balloon-catheter system, under fluoroscopic guidance. The proximal catheter, either a 4 French SwanGanz monitoring catheter (American Edwards Laboratories, Anasco, Puerto Rico) for the normal artery studies or a $2.0-$ or $2.5-\mathrm{mm}$ balloon angioplasty catheter (Mansfield Scientific, subdivision of Boston Scientific, Watertown, MA) for the atherosclerotic artery studies, was introduced through the right common carotid artery. A baseline angiogram of the aorta and iliac branches was obtained pretransfection, after which, the tip of the catheter was positioned at the origin of the left external iliac artery. The distal catheter, a 2 French Fogarty embolectomy catheter (American Edwards Laboratories), was advanced in retrograde fashion through the femoral artery, and its balloon positioned $1.0-1.5 \mathrm{~cm}$ distal to the tip of the more proximally positioned catheter; the distance between the two balloons thus defined the $1.0-1.5-\mathrm{cm}$ long segment of external iliac artery to be isolated upon dual balloon inflation. In the atherosclerotic animals, a supplemental step, balloon angioplasty, was performed on the lesion before the transfection. This was necessary to create an adequately sized intraluminal chamber for the delivery of the full volume of DNA-liposome solution at the lesion site. Furthermore, this fully recreated the clinical scenario of the balloon-injured atherosclerotic lesion likely to represent one potential application of vascular gene transfer therapy.

After verifying the position of the two balloons, the proximal one was inflated, and the distal vessel washed sequentially with normal saline $(5 \mathrm{ml}$ ) and reduced-serum medium (Opti-MEM) ( $5 \mathrm{ml}$ ). Next, the DNA-liposome solution was infused through the same catheter lumen and the distal balloon completely inflated near the end of the perfusion to isolate the arterial segment from serum proteins. The two

nal iliac artery. $(b)$ Positioning of dual catheter system during percutaneous transfection. Arrows indicate proximal and distal extent of isolated transfection compartment within left external iliac artery. The proximal catheter in this case is a 4 French Swan-Ganz monitoring catheter and the distal one a 2 French Fogarty embolectomy catheter. $(c)$ Infusion of contrast media through the proximal catheter delineates the borders of the transfection chamber, again outlined by the arrows. 
Table I. Results of Luciferase Activity Assay in Cultured Arterial Segments Transfected In Vitro

\begin{tabular}{cc}
\hline DNA-liposome ratio $(w t / w t)$ & TLU (mean \pm SEM)* \\
\hline $1: 1$ & $2.27 \pm 1.39$ \\
$1: 2$ & $18.03 \pm 6.46$ \\
$1: 3$ & $26.40 \pm 7.93$ \\
$1: 4$ & $34.75 \pm 11.53$ \\
$1: 5$ & $28.31 \pm 11.75$
\end{tabular}

* From 20- $\mu$ l aliquot out of $250-\mu 1$ sample.

balloons remained inflated for a period of $30 \mathrm{~min}$ and were then deflated. Only one arterial segment per animal was transfected with pRSVLUC to minimize the possibility of remote gene transfection. Controls were obtained using the untreated artery from the contralateral limb in all 10 normal rabbits, and 9 of 12 atherosclerotic rabbits; in the remaining 3 atherosclerotic animals, the contralateral control artery was also treated by balloon angioplasty, but transfected with a DNA-liposome solution composed of a luciferase-less and promoterless plasmid (pGEM3, Promega Corp.) and Lipofectin, as described above. All rabbits were electively killed 3-21 d after the procedure.

Tissue harvesting, analysis of luciferase reporter gene activity, and controls. Monitoring of luciferase activity was done using the Luciferase Assay System (Promega Corp.). Briefly, the artery to be analyzed was ground to a powder, resuspended at room temperature in $1 \times$ cell culture lysis reagent and homogenized (Virtis Co., Inc., Gardiner, NY). The resulting mixture was spun for 5 min to pellet large debris. A 20- $\mu$ l aliquot of the cell extract was then mixed in a sample tube with $100 \mu \mathrm{l}$ of Luciferase Assay Reagent at room temperature and inserted into a luminometer (Model 20e; Turner Designs, Sunnyvale, CA) that reports results on a scale established to yield as low as $10^{-3}$ Turner light units (TLU) ${ }^{1}$. Three different aliquots for each specimen were analyzed in this manner. The specimen's total luciferase activity was derived from the mean of the aliquot results. The light unit values obtained were within the linear range of a dilution curve established by analysis of light units produced from a known amount of luciferase (Sigma Chemical Co., St. Louis, MO). Although assays of different dilutions of luciferase standards constantly yielded linear curves, conversion into picograms resulted in important level variations depending on the source of the standard, as others have also reported (18). Thus, results are expressed as Turner light units. As a reference, under our conditions, transfection of rabbit vascular smooth muscle cells ( $80 \%$ confluent) in $60-\mathrm{mm}$ dishes, for $30 \mathrm{~min}$, using $15 \mu \mathrm{g}$ of pRSVLUC and $45 \mu \mathrm{g}$ of Lipofectin, yielded $431.25 \pm 49.13$ TLU per dish (total from all cells, five dishes) when assayed $3 \mathrm{~d}$ later. Background level was always measured by analyzing a sample consisting only of cell culture lysis reagent and luciferase assay reagent but lacking homogenized tissue; all such analyses consistently produced readings of zero light units.

In situ hybridization of transfected artery. To localize and determine the number of cells successfully transfected during the in vivo procedure, sections from a mid-portion of an atherosclerotic artery transfected with pRSVLUC (6.06 TLU on luciferase assay) and controlled with a contralateral arterial transfection of pGEM3 (no TLU on luciferase assay) were hybridized with antisense and sense-radiolabeled probes generated from a riboprobe vector, pOLUC, containing the same firefly luciferase cDNA as that found in pRSVLUC. The pGEM3 transfected artery served as negative tissue control to the luciferase antisense probe. Also, to rule out the probability that our probes would in fact detect the transfected DNA copies of pRSVLUC, sections from both arteries were also hybridized with radiolabeled antisense and sense RNA probes transcribed from pGEM3. Probe preparation and in situ

1. Abbreviation used in this paper: TLU, Turner light units. hybridization procedure were performed according to our previously reported protocol (19). Autoradiography slides were developed after an exposure time of $10 \mathrm{~d}$.

Angiographic analysis. Angiograms of the transfected segment of the external iliac artery, obtained before and after angioplasty, were recorded on $105-\mathrm{mm}$ spot films before transfection of the genetic material. The angiograms were digitized on a laser film digitizer ( Laser Scan; ImageComm, Santa Clara, CA) and lesions quantified using a previously validated (20) edge-detection software program (Quantum 2000i; QCS, Ann Arbor, MI). Angiographic luminal diameter narrowing, expressed as percent stenosis, was determined by comparison with the largest lumen diameter distal to the lesion. The transfected segment was analyzed for percent luminal narrowing at the site of most severe focal stenosis, as well as for mean percent luminal narrowing along the entire length of the transfected segment.

Morphometric analysis. A 5-mm-long segment of artery, beginning immediately contiguous to the distal-most site of the transfected arterial segment, was also retrieved for histologic, including morphometric, examination. Sections were prepared from six levels of each of these 5-mm-long segments. Each section was stained with hematoxylin-eosin and Richardson's elastic-trichrome stains. The thickness of atherosclerotic plaque was measured at 15 equally distributed sites on each section using a graphics digitizing table (Summasketch; Summagraphics Corp., Seymour, CT); for each specimen the results are expressed as a mean of the six sections for that specimen. Because the treated (transfected) arterial segments were retrieved at necropsy and processed for analysis of luciferase activity, pressure-perfusion-fixation with formalin before retrieval was omitted to preserve the validity of measurements made in the luminometer.

Statistical analysis. Results are expressed as mean \pm SEM. Statistical differences regarding organ culture transfections and percent stenosis before and after angioplasty were determined using, respectively, an unpaired and paired two-tailed Student's $t$ test. Outcome of the transfection procedures in normal and atherosclerotic animals was compared by chi-square analysis. Significance levels regarding differences in luciferase activity levels and angiographic results were assessed by using analysis of variance followed by Sheffe's test. Differences were considered statistically significant if $P<0.05$.

\section{Results}

Arterial organ culture transfections (Table I). Five different ratios of DNA to liposomes were tested in vitro using an arterial organ culture system. The transfection period was restricted to $30 \mathrm{~min}$ to reflect the in vivo application. Analysis of the transfected organs revealed successful gene transfer in every

Table II. Results of Luciferase Activity Assay in Normal Arteries after Percutaneous Transfection

$\begin{array}{cccc}\begin{array}{c}\text { Normal } \\ \text { rabbit \# }\end{array} & \begin{array}{c}\text { Treated segment: } \\ \text { total TLU }\end{array} & \begin{array}{c}\text { Control segment: } \\ \text { total TLU }\end{array} & \begin{array}{c}\text { Sacrifice after } \\ \text { transfection }\end{array} \\ & & & d \\ 1 & 2.39 & 0 & 3 \\ 2 & 0 & 0 & 3 \\ 3 & 5.05 & 0 & 5 \\ 4 & 0 & 0 & 5 \\ 5 & 0 & 0 & 6 \\ 6 & 0 & 0 & 6 \\ 7 & 0 & 0 & 7 \\ 8 & 0 & 0 & 7 \\ 9 & 4.65 & 0 & 14 \\ 10 & 0.04 & 0 & 21\end{array}$


Table III. Results of Luciferase Activity Assay after Percutaneous Transfection in Balloon-dilated Atherosclerotic Arteries

\begin{tabular}{cccc}
$\begin{array}{c}\text { Atherosclerotic } \\
\text { rabbit \# }\end{array}$ & $\begin{array}{c}\text { Treated segment: } \\
\text { total TLU }\end{array}$ & $\begin{array}{c}\text { Control segment: } \\
\text { total TLU }\end{array}$ & $\begin{array}{c}\text { Sacrifice after } \\
\text { transfection }\end{array}$ \\
\hline & & & $d$ \\
1 & 0 & 0 & 6 \\
2 & 0 & 0 & 5 \\
3 & 5.14 & 0 & 4 \\
4 & 12.45 & 0 & 4 \\
5 & 5.03 & 0 & 3 \\
6 & 0 & 0 & 6 \\
7 & 0 & 0 & 7 \\
8 & 0 & 0 & 6 \\
9 & 0 & 0 & 7 \\
10 & 0.10 & 0 & 5 \\
11 & 6.06 & 0 & 5 \\
12 & 0.07 & 0 & 5 \\
\end{tabular}

segment. A broad range of DNA to liposomes ratios proved to yield optimal transfection efficiencies (1:2-1:5), as no significant difference in results could be found between these four formulations. Only a ratio of $1: 1$ resulted in efficiency that was significantly lower than $1: 2(P=0.03), 1: 3(P=0.02)$, and $1: 4$ $(P=0.03)$.

Luciferase activity in arterial segments transfected in vivo (Tables II and III). Percutaneous, site-specific intraarterial gene transfer was attempted in a total of 22 arterial segments from 22 animals. Transfected and contralateral control segments were successfully retrieved after a mean $6.4 \mathrm{~d}$ (3-21 d) after gene transfer. Luciferase activity for each of the transfected segments is shown in Table II (normal) and Table III (balloon-injured atherosclerotic). Of the 22 segments transfected with the pRSVLUC plasmid, $10(45.5 \%)$ showed luciferase activity when assayed in the luminometer. Analysis of the
10 transfected normal segments, examined at a mean $7.7 \mathrm{~d}$ (3-21 d) after procedure, resulted in light detection in four (40\%) specimens. Rabbits killed at both 14 and $21 \mathrm{~d}$ after transfection were part of the group that yielded positive results. Among the 12 balloon-injured atherosclerotic arteries, examined at a mean 5.3 d (3-7 d) after transfer, luciferase activity was documented in $6(50 \%)$. Frequency of successful transfection, as determined by the number of segments where luciferase activity was detected per number of attempted procedures, was not significantly different when the two groups were compared.

The mean value of luciferase activity for all successfully transfected segments was 4.10 \pm 1.19 TLU. Among those, normal arterial segments yielded a mean $3.03 \pm 1.16$ TLU $(0.04-$ 5.05 TLU) per specimen, whereas the six balloon-injured atherosclerotic arteries transfected with pRSVLUC had a mean level of light emission of $4.81 \pm 1.87$ TLU (0.07-12.45 TLU). When compared with the normal artery transfection results, there was no significant difference in luciferase activity levels obtained in the atherosclerotic animals.

The untreated external iliac artery of the contralateral limb from 19 rabbits was retrieved and analyzed for luminescence as a control. None of these specimens disclosed any evidence of luciferase activity. In three atherosclerotic animals, we repeated the balloon dilatation procedure in the external iliac artery of the contralateral limb, after which a plasmid (pGEM3) lacking both the luciferase gene and the Rous sarcoma virus long terminal repeat promoter was transfected. This was done as a control for the luciferase assays as well as for the in situ hybridization study. Again, all of these yielded zero TLU when assayed for luciferase activity.

Angiographic analysis of transfected atherosclerotic arterial segments (Table IV). Results of quantitative angiographic lesion analysis are presented in Table IV. The severity of lesions was assessed by measuring the angiographically derived percent stenosis of the angioplasty site, before and after angioplasty; the transfection procedure was performed at precisely

Table IV. Angiographic and Pathologic Findings in Percutaneously Transfected Atherosclerotic Arterial Segments

\begin{tabular}{|c|c|c|c|c|c|c|}
\hline \multirow[b]{2}{*}{ Rabbit \# } & \multicolumn{4}{|c|}{ Angiographic findings } & \multicolumn{2}{|c|}{ Pathologic findings } \\
\hline & $\begin{array}{l}\text { Maximal \% stenosis of } \\
\text { transfected segments } \\
\text { before PTA }\end{array}$ & $\begin{array}{l}\text { Maximal \% stenosis of } \\
\text { transfected segments } \\
\text { after PTA }\end{array}$ & $\begin{array}{c}\text { Mean \% stenosis of } \\
\text { transfected segments } \\
\text { before PTA }\end{array}$ & $\begin{array}{l}\text { Mean \% stenosis of } \\
\text { transfected segments } \\
\text { after PTA }\end{array}$ & $\begin{array}{l}\text { Mean neointimal thickness } \\
\text { immediately contiguous to } \\
\text { treated segment } \mathrm{m} \pm \mathrm{SEM}\end{array}$ & $\begin{array}{l}\text { \% Lumen circumference } \\
\text { covered by plaque in } \\
\text { immediately contiguous } \\
\text { segments }\end{array}$ \\
\hline & & & & & $\mu m$ & \\
\hline 1 & 64 & 49 & 29 & 17 & $134 \pm 8$ & 100 \\
\hline 2 & 45 & 39 & 15 & 3 & $182 \pm 12$ & 100 \\
\hline $3^{*}$ & 39 & 30 & 13 & 32 & $256 \pm 22$ & 98 \\
\hline $4^{*}$ & 40 & 31 & 34 & 27 & $245 \pm 12$ & 100 \\
\hline $5^{*}$ & 63 & 42 & 25 & 22 & $276 \pm 23$ & 99 \\
\hline 6 & 40 & 46 & 34 & 13 & $428 \pm 33$ & 100 \\
\hline 7 & 39 & 20 & 10 & 12 & $233 \pm 17$ & 100 \\
\hline 8 & 34 & 53 & 25 & 28 & $594 \pm 21$ & 100 \\
\hline 9 & 35 & 17 & 12 & 10 & $377 \pm 14$ & 100 \\
\hline $10^{*}$ & 40 & 46 & 19 & 22 & $227 \pm 21$ & 100 \\
\hline $11^{*}$ & 75 & 39 & 43 & 15 & $336 \pm 18$ & 100 \\
\hline $12^{*}$ & 57 & 28 & 31 & 7 & $360 \pm 16$ & 100 \\
\hline
\end{tabular}

* Successfully transfected; PTA, percutaneous transluminal angioplasty. 

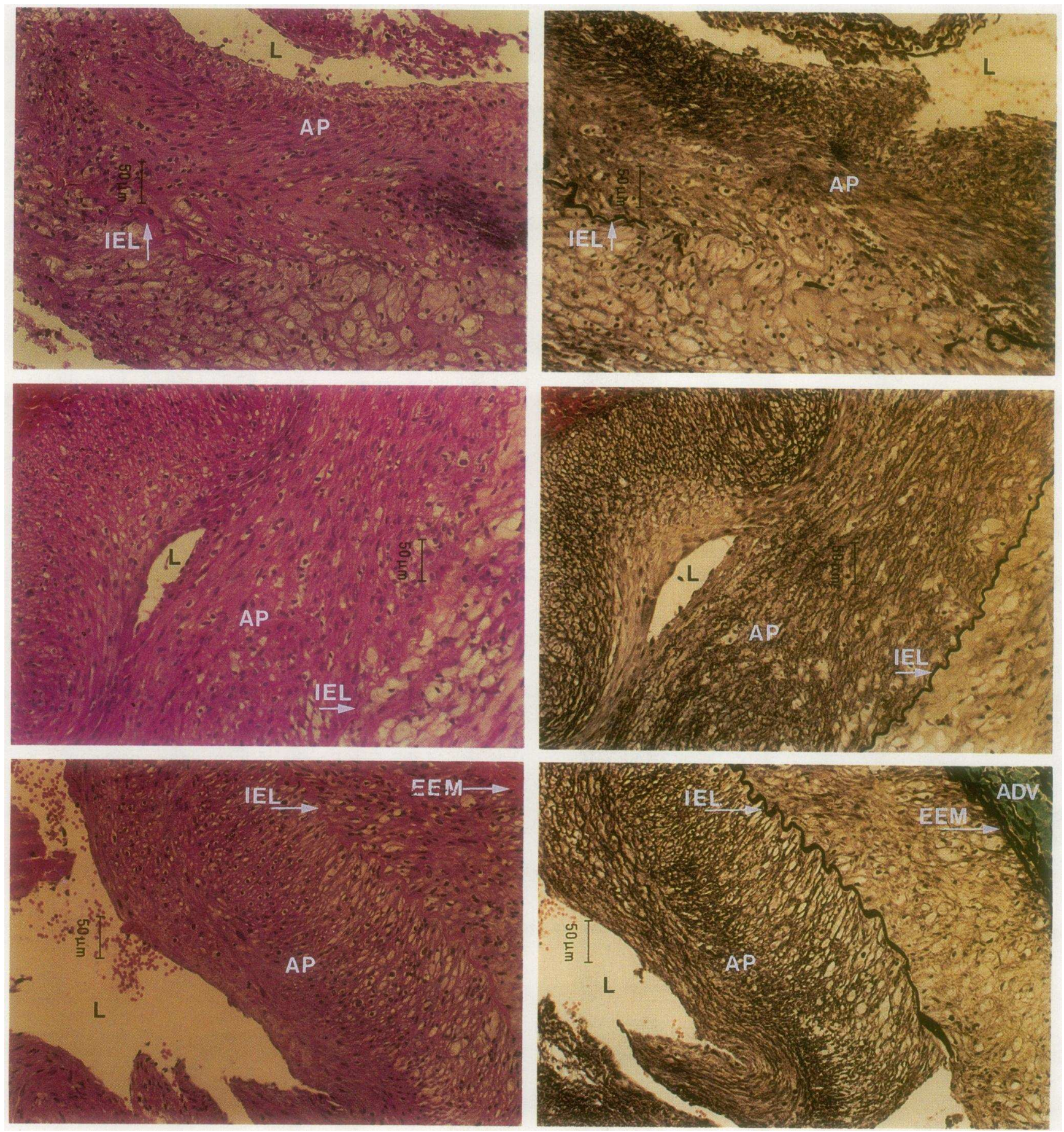

Figure 2. Representative light microscopic findings in post-angioplasty, transfected atherosclerotic artery from rabbits \#3 (top), 4 ( $m i d d l e$ ), and 5 (bottom). (IEL, internal elastic membrane; EEM, external elastic membrane; ADV, adventitia; AP, atherosclerotic plaque; L, residual lumen). Left, hematoxylin and eosin; right, elastic-tissue trichrome stain.

this site. Angiographic analysis may have underestimated the degree of luminal narrowing in the transfected segments since this was expressed as a percentage of the luminal diameter of the distal segment, which itself was balloon-denuded and documented at necropsy (vide infra) to be narrowed by atherosclerotic plaque.

Two types of quantitative angiographic analyses were performed. First, the site of most severe focal stenosis in the transfected segment of the artery was assessed. Maximal focal steno- sis before and after dilatation were $48 \pm 4 \%$ and $37 \pm 3 \%$, respectively, resulting in a statistically significant difference ( $P$ $=0.03$ ). Second, the mean stenosis of the entire length of the transfected segment was measured. Mean before and after dilatation were $23 \pm 3 \%$ and $17 \pm 3 \%$, respectively $(P=\mathrm{NS})$.

Morphologic analysis of transfected atherosclerotic arterial segments (Fig. 2, Table IV). Histologic examination disclosed a highly cellular neointimal lesion, with abundant foam cells in both treated and control arterial segments. This proliferative 

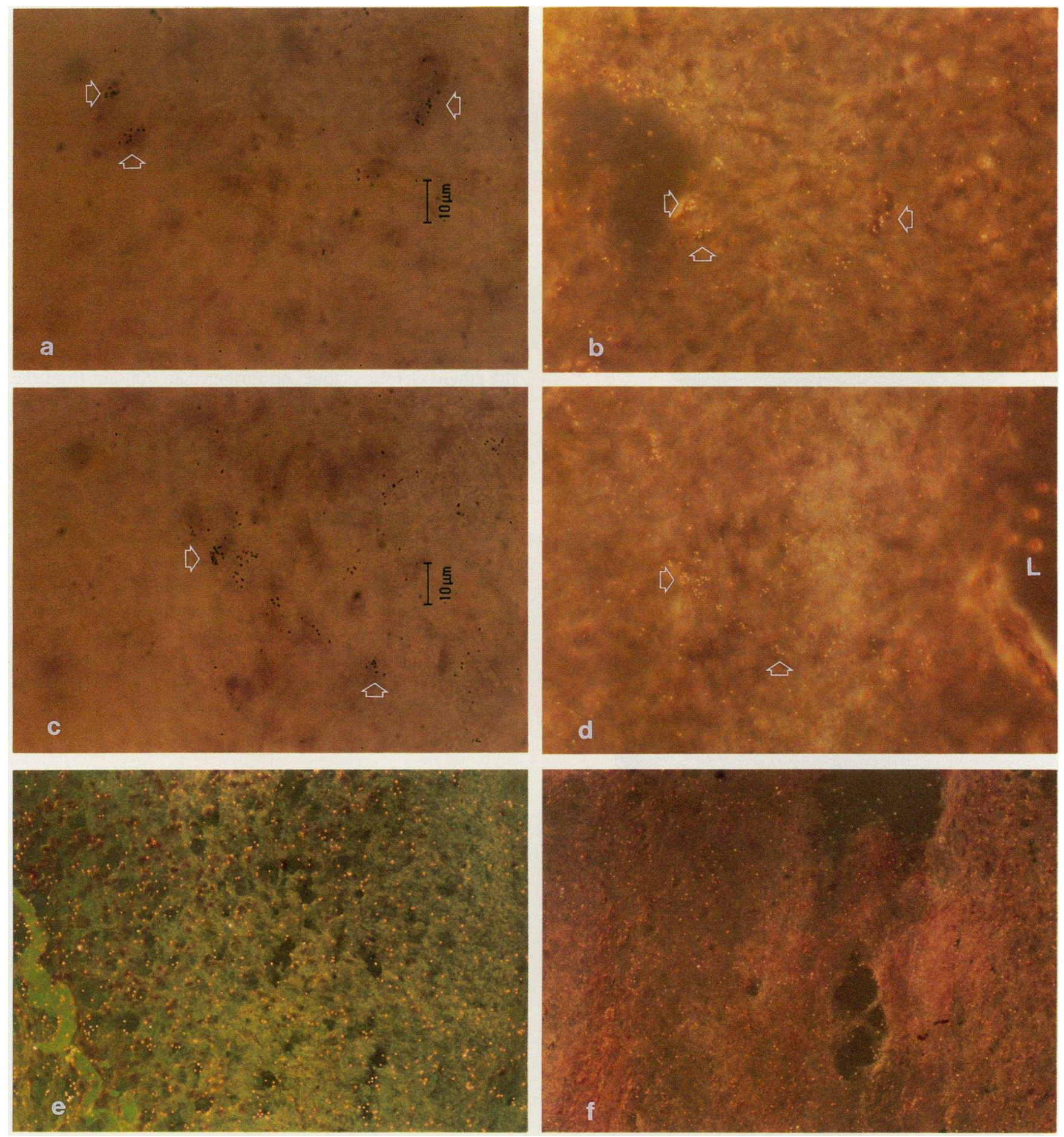

Figure 3. In situ hybridization of frozen sections from transfected balloon-dilated atherosclerotic artery (treatment and control artery, rabbit 11, Table III). $(a)$ and $(c)$ High power bright-field photomicrograph of different fields from a section transfected with pRSVLUC and exposed to the antisense radiolabeled probe generated from pOLUC. Arrows indicate cells, recognized by the presence of nucleus, associated with a cluster of dark silver grains, indicating presence of a positive hybridization signal. $(b)$ and $(d)$ Dark-field photomicrograph of the same section and probe as in $(a)$ and $(c)$, respectively. Arrows indicate location of cells outlined in $(a)$ and $(c)$, at lower magnification. L, lumen, (photographed at $150 \times) .(e)$ Dark-field photomicrograph of tissue section transfected with pRSVLUC and exposed to the sense radiolabeled probe generated from pOLUC. No hybridization signal similar to that seen in the above mentioned section could be detected (photographed at $\times 75)$. $(f)$ Darkfield photomicrograph of a section transfected with pRSVLUC but exposed to the antisense radiolabeled probe generated from pGEM3 to rule out the possibility that the hybridization signal was from the foreign DNA template originally transfected. Again no signal was found (photographed at $\times 75)$. 


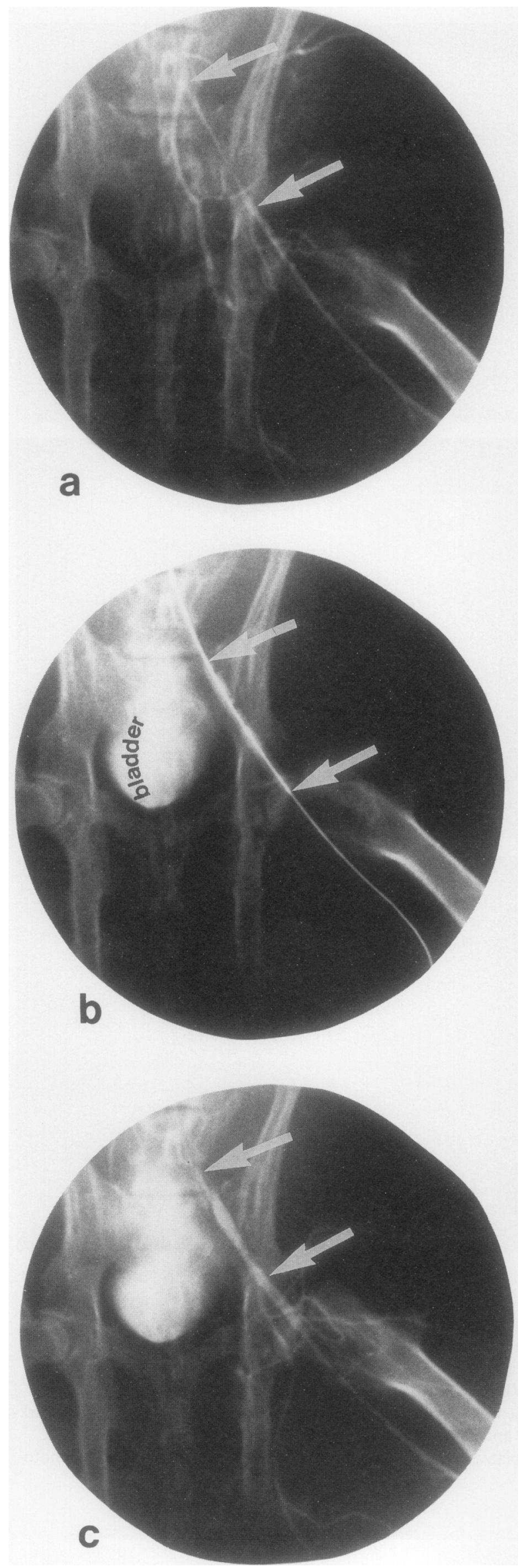

process covered $\geq 98 \%$ of the total circumference of the arterial lumen in each case.

Morphometric analysis of light microscopic sections was performed to establish quantitatively the status of the arterial wall into which the new genetic information was to be transfected. Measurements of neointimal thickness were performed at six different levels of each section retrieved at necropsy. Mean neointimal thickness of all treated (transfected) segments was $304 \pm 35.9 \mu \mathrm{m}(134-594 \mu \mathrm{m})$.

In situ hybridization of a transfected vessel (Fig. 3). Results of the in situ hybridization performed on the successfully transfected atherosclerotic arterial sections are presented in Fig. 3. Sections exposed to the antisense luciferase probe generated from pOLUC showed that only a few cells in the transfected atherosclerotic arterial segment were responsible for luciferase expression. Cells showing hybridization were located within the atherosclerotic plaque, no further than $200 \mu \mathrm{m}$ from the lumen where the DNA-liposomes solution was infused. In total, $<1 / 1,000$ cells could be identified as showing a hybridization pattern (Fig. 3, $a-d$ ). Furthermore, cells identified as expressing the luciferase mRNA were usually found in groups of six to seven cells separated by $100 \mu \mathrm{m}$ between each other. Sections exposed to the luciferase sense probe revealed no evidence of hybridization (Fig. $3 e$ ). As a negative tissue control, we exposed the luciferase antisense probe to sections of the contralateral atherosclerotic artery transfected with pGEM3. Again, no positive signal was detected on these slides. Finally, we hybridized antisense probes generated from the pGEM plasmid on the luciferase transfected tissue to rule out the possibility that we were in fact detecting copies of the transfected DNA. None of these sections revealed signs of positive hybridization (Fig. $3 f$ ).

\section{Discussion}

The limitations of purely mechanical techniques in diminishing the incidence of restenosis after percutaneous revascularization have prompted investigation of genetic engineering as a potential adjunct for catheter-based interventions. The feasibility of this approach requires demonstration that genetic material can be successfully transferred to the arterial wall at the site of intended intervention. This prerequisite was successfully established in an elegant series of experiments performed by Nabel and co-workers $(6,8,9)$. These investigators, using the lac $Z$ reporter gene responsible for $\beta$-galactosidase production, accomplished both indirect as well as direct transfection of surgically exposed normal iliofemoral swine arteries. The former involved delivery of both endothelial (8) and vascular smooth muscle cells $(9)$ infected with a replication-defective retroviral

Figure 4. Left external iliac artery angiogram of an atherosclerotic animal. (a) Preangioplasty angiogram of a previously denuded external iliac artery showing diffuse and severe arterial lumen diameter reduction ( $75 \%$, maximal stenosis) between the origin of the internal iliac artery (top arrow) and the first subsequent side branch (lower arrow). (b) Balloon-angioplasty of targeted arterial site to create chamber of sufficient size to accomodate DNA-liposome solution. Arrows, in this case, indicate extent of the inflated balloon. (c) Postangioplasty angiogram of the same artery, demonstrating improvement in luminal size, thus enabling infusion of solution within targeted space (between arrows). 
vector, whereas direct transfection was achieved with both the retroviral vector and cationic liposomes (6). Indirect transfection was also accomplished by Wilson et al. in dogs using an implanted carotid interposition prosthetic graft seeded with genetically engineered endothelial cells (10). More recently, Lim et al. (7) reported direct liposome-mediated transfer of the luciferase reported gene to surgically exposed normal canine coronary and peripheral arteries.

The current experiments extend these previous findings by examining the efficiency of an exclusively percutaneous sitespecific delivery of DNA to normal and balloon-dilated atherosclerotic arteries; the latter represents a potential clinical strategy for the prevention of restenosis. Our results show that sitespecific gene transfer can be successfully accomplished by such an exclusively percutaneous approach in $45 \%$ of cases. This contrasts with in vitro transfection efficiency, where liposomemediated gene transfer of rabbit arterial organ culture, performed under the same ratio conditions, results in expression of the reporter gene in $100 \%$ of cases. The current data also must be compared to results reported by Lim et al. (7) who detected luciferase activity in 9 of $10(90 \%)$ segments transfected in vivo in a surgically controlled environment. In their protocol, the DNA-liposome solution (used with the same DNA-Lipofectin ratio as current protocol) was infused under direct vision after exposing the arteries and securing the transfected arterial chamber with a removable ligature. Although the frequency of deliveries leading to detectable luciferase activity appears to be lower with the current approach, the levels of reporter gene expression in successfully transfected animals are comparable. Not withstanding the inherent difficulty of accurately converting light units to amounts of luciferase protein present (18), our results reflect the presence of luciferase enzyme in the range of at least $10^{-12} \mathrm{~g}$ per successfully treated artery, a range similar to that found in transfected arteries of comparable size reported by others (7).

Several factors may account for the lower transfection efficiency observed in our experiments. First, while a DNA-liposome ratio of 1:3 has been successfully used in other species, it is conceivable that this ratio may be suboptimal in the rabbit model. In vitro transfection of cultured rabbit arterial organs, however, indicated that the ratio of 1:3 was part of a group of ratios that resulted in the highest levels of transfection. This broad range of effective ratios has also been reported by others in different types of tissues (21). A second potential basis for the low transfection efficiency is inadvertent mixing of the DNA-liposome solution with serum proteins, a factor known to reduce the transfection efficiency with Lipofectin (14). Such contamination might have occurred, for example, due to insufficient sealing of the chamber by the inflated balloons. Third, undetected leakage of the solution through unrecognized side branches might also reduce the volume of solution being infused into the arterial transfection chamber demarcated between the two inflated balloons.

We used the firefly reporter gene for our experiments because of high sensitivity, absence of background activity as measured under our conditions, and the quantifiable nature of the assay $(11,12)$. We have previously investigated use of the $\beta$-galactosidase-producing lac $Z$ reporter gene to monitor sitespecific transfection in nonatherosclerotic New Zealand white rabbits (unpublished data). In contrast to the previously published experience with this reporter system in the swine model (6), the finding of endogenous $\beta$-galactosidase activity in sham-treated arteries forced us to conclude that this reporter system was not suitable for the rabbit model.

Our transfection procedures were performed in two types of environment, normal and balloon-dilated atherosclerotic arteries. Transfection of the latter was performed to simulate the anticipated strategy of coupling percutaneous gene transfer to balloon angioplasty in an attempt to inhibit restenosis. Furthermore, balloon inflation was essential in the current protocol to create in each animal a chamber of sufficient volume to accomodate the DNA-liposome solution at the targeted arterial site (Fig. 4). Frequency of transfection as well as levels of luciferase activity obtained in normal and balloon-dilated atherosclerotic arteries were similar. The extent of atherosclerotic narrowing was demonstrated angiographically and histologically. The angiographic measurements, in fact, underestimated the severity of the lesions, since percent narrowing of the transfected segment was calculated as a percentage of the largest distal luminal dimeter and this distal segment was itself narrowed by atherosclerotic plaque. The severity of the atherosclerotic lesions was such that percutaneous delivery, under fluoroscopic guidance, was probably the method choice for the purpose of ensuring infusion specifically within the lumen of the diseased artery. Morphometric analysis of these segments confirmed that the atherosclerotic plaque separated the entire circumference of the native arterial wall from the residual arterial lumen (Table IV) except in two where it covered 98 and $99 \%$ of the lumen. In situ hybridization documented that successful gene transfer had been achieved in the diseased layers of the atherosclerotic arterial segments.

In situ hybridization has been used by others to localize site of luciferase mRNA expression in transfected tissues (22). Our studies were performed on sections of a transfected balloon-dilated atherosclerotic artery showing a significant lesion, which is reported to be composed mainly by smooth muscle cells (23). They disclosed a low efficiency resulting from an exclusively percutaneous delivery strategy. The hybridization signal found in the histologic sections exposed to the pOLUC antisense probe demonstrates that transgenes in relatively few cells account for the levels of luciferase activity indicated by the luminometer readings. Moreover, these cells appeared to be located within $200 \mu \mathrm{m}$ of the lumen border where the solution was infused.

This study thus demonstrates that expression of new genetic material may be achieved in both normal and balloon-injured atherosclerotic arteries following an exclusively percutaneous approach. The low efficiency of the current delivery strategy, however, remains an important challenge. Logistic configuration of the percutaneous approach as well as the in vivo biologic behavior of the courier mediating the gene transfer will need to be improved if this strategy is to eventually be applied as a therapeutic approach to human vascular disease.

\section{Acknowledgments}

The authors gratefully acknowledge the assistance of Eleanor M. Sullivan and Marianne Kearney in the processing and preparation of slides for light-microscopic analysis; John Hogan in performing the quantitative histologic measurements; Jenifer K. Daly in performing the quantitative angiographic analyses; and Mickey Ratti in the preparation of this manuscript. Dr. Allen Brasier generously supplied the plasmid constructs used in this investigation. 
This work was supported in part by grants from the National Heart, Lung, and Blood Institute (grants HL-40518 [J. M. Isner] and R9AR40580A [L. Weir]), Bethesda, MD. Dr. Leclerc is a Research Fellow of the Heart and Stroke Foundation of Canada. Dr. Nikol is the recipient of an award from the Deutsche Forschungsgemeinschaft.

\section{References}

1. Kitsis, R. N., P. M. Buttrick, E. M. McNally, M. L. Kaplan, and L. A. Leinwald. 1991. Hormonal modulation of a gene injected into rat heart in vivo. Proc. Natl. Acad. Sci. USA. 88:4138-4142.

2. Rosenberg, S. A., P. Aebersold, K. Cornetta, A. Kasid, R. A. Morgan, R. Moen, E. M. Karson, M. T. Lotze, J. C. Yang, S. L. Topalian, et al. 1990. Gene transfer into humans. Immunotherapy of patients with advanced melanoma, using tumor-infiltrating lymphocytes modified by retroviral gene transduction. N. Engl. J. Med. 323:570-578.

3. Anderson, W. F., R. M. Blaese, and K. Culver. 1990. The ADA human gene therapy clinical protocol. Hum. Gene Ther. 1:331-362.

4. Culliton, B. J. 1991. Gene therapy on the move. Nature (Lond.). 354:429.

5. McBride, W., R. Lange, and L. Hillis. 1988. Restenosis after successful coronary angioplasty. N. Engl. J. Med. 318:1734-1737.

6. Nabel, E. G., G. Plautz, and G. J. Nabel. 1990. Site-specific expression in vivo by direct gene transfer into the arterial wall. Science (Wash. DC). 249:12851288.

7. Lim, C. S., G. D. Chapman, R. S. Gammon, J. B. Mulhestein, R. P. Bauman, R. S. Stack, and J. L. Swain. 1991. Direct in vivo gene transfer into the coronary and peripheral vasculatures of the intact dog. Circulation. 83:20072011.

8. Nabel, E. G., G. Plautz, F. M. Boyce, J. C. Stanley, and G. J. Nabel. 1989. Recombinant gene expression in vivo within endothelial cells of the arterial wall. Science (Wash. DC). 244:1342-1344.

9. Plautz, G., E. G. Nabel, and G. J. Nabel. 1991. Introduction of vascular smooth muscle cells expressing recombinant genes in vivo. Circulation. 83:578583.

10. Wilson, J. M., L. K. Birinyi, R. N. Salomon, P. Libby, A. D. Callow, and R. C. Mulligan. 1989. Implantation of vascular grafts lined with genetically modified endothelial cells. Science (Wash. DC). 244:1344-1346.
11. de Wet, J. R., K. V. Wood, M. DeLuca, D. R. Helinski, and S. Subramani. 1987. Firefly luciferase gene: structure and expression in mammalian cells. $\mathrm{Mol}$. Cell. Biol. 7:725-737.

12. Brasier, A. R., J. E. Tate, and J. F. Habener. 1989. Optimized use of the firefly luciferase assay as a reporter gene in mammalian cell lines. Biotechniques. 7:1116-1122.

13. Felgner, P. L., T. R. Gadek, M. Holm, R. Roman, H. D. Chan, M. Wenz, J. P. Northrop, G. M. Ringold, and M. Danielsen. 1987. Lipofection: a highly efficient, lipid-mediated DNA-transfection procedure. Proc. Natl. Acad. Sci. USA. 84:7413-7417.

14. Felgner, P. L., and G. M. Ringold. 1989. Cationic liposome-mediated transfection. Nature (Lond.). 337:387-388.

15. Todd, M. E., and S. M. Friedman. 1978. The rat-tail artery maintained in culture: an experimental model. In Vitro (Rockville). 14:757-770.

16. Merrilees, M., and L. Scott. 1982. Organ culture of rat carotid artery: maintenance of morphological characteristics and of pattern of matrix synthesis. In Vitro (Rockville). 18:900-910.

17. Baumgartner, H. R., and A. Studer. 1963. Controlled over-dilation of the abdominal aorta in normo- and hypercholesterolemic rabbits. Pathol. Microbiol. 26:129-148.

18. Wolff, J. A., P., Williams, G. Acsadi, S. Jiao, A. Jani, and W. Chong. 1991. Conditions affecting direct gene transfer into rodent muscle in vivo. Biotechniques. 11:474-485.

19. Leclerc, G., J. M. Isner, M. Kearney, M. Simons, R. D. Safian, D. S. Baim, and L. Weir. 1992. Evidence implicating nonmuscle myosin in restenosis: use of in situ hybridization to analyze human vascular lesions obtained by directional atherectomy. Circulation. 85:543-553.

20. Sanders, W. J., E. L. Alderman, and D. C. Harrison. 1979. Coronary artery quantitation using digital image processing techniques. IEEE (Inst. Electr. Electron. Eng.) Comput. Card. 7:15-20.

21. Muller, S. R., P. D. Sullivan, D. O. Clegg, and S. C. Feinstein. 1990. Efficient transfection and expression of heterologous genes in PC12 cells. DNA Cell Biol. 9:221-229.

22. Williams, R. S., S. A. Johnston, M. Riedy, M. J. DeVit, S. G. McElligott, and J. C. Sanford. 1991. Introduction of foreign genes into tissues of living mice by DNA-coated microprojectiles. Proc. Natl. Acad. Sci. USA. 88:2726-2730.

23. Weidinger, F. F., J. M. McLenachan, M. I. Cybulski, J. T. Fallon, N. K. Holloenburg, J. P. Cooke, and P. Ganz. 1991. Hypercholesterolemia enhances macrophage recruitment and dysfunction of regenerated endothelium after balloon injury of the rabbit iliac artery. Circulation. 84:755-767. 